ANNALES

POLONICI MATHEMATICI

$86.1(2005)$

\title{
The Jacobian Conjecture for symmetric Drużkowski mappings
}

\author{
by Michiel De Bondt and ARno VAn DEN Essen (Nijmegen)
}

\begin{abstract}
Let $k$ be an algebraically closed field of characteristic zero and $F:=$ $x+(A x)^{* d}: k^{n} \rightarrow k^{n}$ a Drużkowski mapping of degree $\geq 2$ with $\operatorname{det} J F=1$. We classify all such mappings whose Jacobian matrix $J F$ is symmetric. It follows that the Jacobian Conjecture holds for these mappings.
\end{abstract}

Introduction. The Jacobian Conjecture asserts that every polynomial map $F: k^{n} \rightarrow k^{n}$ with $\operatorname{det} J F \in k^{*}$ is invertible. In the classical papers [1] and [4] it is shown that it suffices to investigate this conjecture for polynomial mappings of the form $x+H$, where $H$ is homogeneous of degree 3 . This result was improved a little later in the paper [3] of Drużkowski: he showed that one may even assume that each component of $H$ is a third power of a linear form. More recently another type of reduction theorem was obtained by the authors in [2]. They showed that it suffices to investigate the Jacobian Conjecture for polynomial mappings of the form $x+H$ with $H$ homogeneous of degree 3 and $J H$ symmetric. This raises the question: does the Jacobian Conjecture hold for Drużkowski mappings with symmetric Jacobian matrix?

The main result of this paper gives an affirmative answer to this question. More precisely we completely classify all these mappings (see Theorem 1 below).

1. Notations and the main theorem. Throughout this paper $k$ denotes an algebraically closed field of characteristic zero and $n$ a positive integer. For any $d \geq 2$ and $A \in M_{n}(k)$ the Druzkkowski mapping ( $D$-mapping for short) of degree $d$ associated to $A$ is the mapping given by $F_{A}:=x+(A x)^{* d}$, where $\left(v_{1}, \ldots, v_{n}\right)^{* d}$ denotes the vector $\left(v_{1}^{d}, \ldots, v_{n}^{d}\right)$. The main result of this paper, Theorem 1, classifies all $D$-mappings with invertible and symmetric Jacobian matrix. It turns out that all these mappings are "products" in a sense defined below of the $D$-mappings described in the following lemma (in

2000 Mathematics Subject Classification: 14R15, 14R10.

Key words and phrases: Jacobian Conjecture, Drużkowski mapping. 
which we use $\langle$,$\rangle to denote the standard bilinear form on k^{n}$, i.e. $\langle x, y\rangle=x^{t} y$ for all $x, y \in k^{n}$, and $\nabla f$ to denote the gradient of a polynomial $f$, i.e. $\left.\left(f_{x_{1}}, \ldots, f_{x_{n}}\right)\right)$.

Lemma 1. Let $F=x+(A x)^{* d}$ with $\mathrm{rk} A \leq 1$. Then $J F$ is invertible and symmetric iff $F$ is of the form $x+\nabla f$, where $f=\langle v, x\rangle^{d+1}$ for some $v \in k^{n}$ with $\langle v, v\rangle=0$. In this case $F$ is invertible with inverse $x-\nabla f$.

Proof. Let $A_{i}$ denote the $i$ th row of $A$ and assume that $A$ has rank 1. Then there exist a non-zero vector $a \in k^{n}$ and $\lambda_{i} \in k$ such that $A_{i}=\lambda_{i} a^{T}$ for all $i$. Furthermore

$$
J:=J\left((A x)^{* d}\right)=d\langle a, x\rangle^{d-1} \mu a^{T},
$$

where $\mu=\lambda^{* d}$.

(i) Now assume that $J F$ is symmetric and invertible. Then $J$ is symmetric. Since $\langle a, x\rangle \neq 0$ it follows that $\mu a^{T}$ is symmetric. So $\mu_{i} a_{j}=\mu_{j} a_{i}$ for all $i, j$, i.e. $\mu$ and $a$ are linearly dependent over $k$, say $\mu=c a$ for some $c \in k^{*}$. Since $J$ is also nilpotent (for $J F_{A}$ is invertible) it follows that $\mu a^{T}$ is nilpotent. So in particular $\operatorname{Tr} \mu a^{T}=0$, i.e. $\langle\mu, a\rangle=0$. From $\mu=c a$ with $c \in k^{*}$ we deduce that $\langle a, a\rangle=0$. Finally, observe that

$$
\left((A x)^{* d}\right)_{i}=\lambda_{i}^{d}\langle a, x\rangle^{d}=\mu_{i}\langle a, x\rangle^{d}=c a_{i}\langle a, x\rangle^{d} .
$$

So if we choose $z \in k$ such that $z^{d}=c a_{i} /(d+1)$ and put $v:=z a$, then one easily verifies that $(A x)^{* d}=\nabla\left(\langle v, x\rangle^{d+1}\right)$.

(ii) Conversely, if $F=x+\nabla f$, where $f=\langle v, x\rangle^{d+1}$ and $\langle v, v\rangle=0$, then clearly $F$ is a $D$-mapping of degree $d$ with symmetric Jacobian matrix. Furthermore, $F=x+(d+1)\langle v, x\rangle^{d} v$. So $\langle v, F\rangle=\langle v, x\rangle$ (since $\left.\langle v, v\rangle=0\right)$. Hence if we put $G:=x-\nabla f=x-(d+1)\langle v, x\rangle^{d} v$, then

$$
G(F)=F-(d+1)\langle v, x\rangle^{d} v=x .
$$

So $F$ is invertible with inverse $x-\nabla f$ and hence $J F$ is invertible, which completes the proof.

A polynomial mapping of the form $x+\nabla f$ with $f=\langle v, x\rangle^{d+1}$ and $\langle v, v\rangle=0$ is called of type 1 . To describe how all $D$-mappings with invertible and symmetric Jacobian matrix can be obtained from these mappings, we introduce some terminology.

Let $x=\left(x_{1}, \ldots, x_{n}\right)$ and $y=\left(y_{1}, \ldots, y_{m}\right)$ be two sets of independent variables. We say that a polynomial map $F: k^{n+m} \rightarrow k^{n+m} a d-$ mits a separation in the variables $x$ and $y$ if there exist polynomial mappings $G, H: k^{n+m} \rightarrow k^{n+m}$ of the form $G=\left(G_{1}(x), \ldots, G_{n}(x), y\right)$ and $H=\left(x, H_{1}(y), \ldots, H_{m}(y)\right)$ such that $F=G \circ H(=H \circ G)$. For simplicity we write $F=G(x) \circ H(y)$. In a similar way we can define when a polynomial map $F: k^{n_{1}+\cdots+n_{s}} \rightarrow k^{n_{1}+\cdots+n_{s}}$ admits a separation in a finite set of 
variables $x^{(1)}=\left(x_{1}^{(1)}, \ldots, x_{n_{1}}^{(1)}\right), \ldots, x^{(s)}=\left(x_{1}^{(s)}, \ldots, x_{n_{s}}^{(s)}\right)$. Finally, a permutation map is a linear map corresponding to a permutation matrix. Now we are able to formulate the main result of this paper:

Theorem 1. Let $F=x+(A x)^{* d}$ be a D-mapping of degree $\geq 2$. If $J F$ is invertible and symmetric, then there exists a permutation map $P$ such that $P^{-1} \circ F \circ P$ admits a separation in a finite set of variables, say $F^{(1)}\left(x^{(1)}\right) \circ \cdots \circ F^{(s)}\left(x^{(s)}\right)$, where each $F^{(i)}$ is a D-mapping of type 1 . In particular, the Jacobian Conjecture holds for D-mappings with symmetric Jacobian matrices.

For the proof we need the following lemma:

Lemma 2. Let $d \geq 2, A=\left(a_{i j}\right) \in M_{n}(k)$ and denote by $A_{i}$ the ith row of $A$. Assume furthermore that $J\left((A x)^{* d}\right)$ is symmetric. Then:

(i) $a_{i j}=0$ iff $a_{j i}=0$.

(ii) If $a_{i j} \neq 0$, then $A_{j}=c A_{i}$ for some $c \in k^{*}$.

Proof. Assume $a_{i j} \neq 0$. To prove (i) it suffices to show that $a_{j i} \neq 0$. Therefore observe that since $J\left((A x)^{* d}\right)$ is symmetric we get $\partial_{j}\left(\left(A_{i} x\right)^{d}\right)=$ $\partial_{i}\left(\left(A_{j} x\right)^{d}\right)$. So $d\left(A_{i} x\right)^{d-1} a_{i j}=d\left(A_{j} x\right)^{d-1} a_{j i}$, which implies that $a_{j i} \neq 0$. Furthermore, also (ii) follows from this equality since both $A_{i} x$ and $A_{j} x$ are linear (and hence irreducible) polynomials in the UFD $k[x]$.

Proof of Theorem 1. We proceed by induction on $n$. The case $n=1$ is obvious, so let $n \geq 2$. We may assume that $A \neq 0$, so it has some non-zero row. One easily verifies that if $P$ is a permutation map, then $P^{-1} \circ F_{A} \circ P=$ $F_{P^{-1} A P}$. So by permuting the variables we may assume that the first row of $A$ is non-zero. Put

$$
E:=\left\{i \mid a_{1 i} \neq 0\right\}
$$

We claim that $a_{11} \neq 0$, i.e. $1 \in E$. Otherwise, since $E$ is not empty, there exists $i>1$ such that $a_{1 i} \neq 0$. Then by Lemma 2 also $a_{i 1} \neq 0$ and $A_{1}=c A_{i}$ for some $c \in k^{*}$. Consequently, $a_{11}=c a_{i 1} \neq 0$, a contradiction. So $1 \in E$. Then, again permuting the variables we may assume that $E=\{1, \ldots, r\}$ for some $1 \leq r \leq n$.

If $1 \leq i \leq r$, then $a_{1 i} \neq 0$. So by Lemma $2, A_{i}=c_{i}\left(a_{11}, \ldots, a_{1 r}, 0, \ldots, 0\right)$ for some $c_{i} \neq 0$. In particular, $a_{i j}=0$ and hence $a_{j i}=0$, if $j>r$ and $1 \leq i \leq r$. So we get

$$
\begin{array}{ll}
F_{i}=x_{i}+c_{i}^{d}\left(a_{11} x_{1}+\cdots+a_{1 r} x_{r}\right)^{d} & \text { for all } 1 \leq i \leq r, \\
F_{j}=x_{j}+\left(a_{j(r+1)} x_{r+1}+\cdots+a_{j n}\right)^{d} & \text { for all } j>r .
\end{array}
$$

In case $r=n$ the result follows from Lemma 1 and if $r<n$ the result follows from the induction hypothesis. 
Acknowledgements. The first author is sponsored by NWO, the Dutch Organisation for Scientific Research.

\section{References}

[1] H. Bass, E. Connell and D. Wright, The Jacobian Conjecture: Reduction of degree and formal expansion of the inverse, Bull. Amer. Math. Soc. 7 (1982), 287-330.

[2] M. de Bondt and A. van den Essen, A reduction of the Jacobian Conjecture to the symmetric case, Proc. Amer. Math. Soc. 133 (2005), 2201-2205.

[3] L. Drużkowski, An effective approach to Keller's Jacobian Conjecture, Math. Ann. 264 (1983), 303-313.

[4] A. V. Yagzhev, Keller's problem, Siberian Math. J. 21 (1980), 747-754.

Department of Mathematics

Radboud University of Nijmegen

Postbus 9010

6500 GL Nijmegen, The Netherlands

E-mail: debondt@math.ru.nl

essen@math.ru.nl 